\title{
RAFAEL FERNÁNDEZ CONCHA Y EL IUSNATURALISMO TOMISTA EN CHILE
}

\author{
Raúl Madrid Ramírez*
}

\begin{abstract}
RESUMEN: El autor nos ofrece una semblanza de la vida y obra del jurista chileno Rafael Fernández Concha, filósofo del derecho y teólogo del s. XIX, de gran erudición y talento especulativo. Su aporte a la ciencia del derecho, vertido en sus cuatro obras principales, es reconocido en áreas tales como Derecho Eclesiástico, Enseñanza del Derecho, Derecho Natural y Filosofía del Derecho de inspiración tomista.

Palabras clave: Filosofía del Derecho, ética tomista, Derecho natural.

ABSTRACT: The author presents us the life and works of Chilean lawyer Rafael Fernandez Concha, who was both a philosopher and a theologist from the nineteenth century, and a man of outstanding erudition and speculative talent. His contribution to the law, as enclosed in his four most important books, is well-known in Church Law, Law Education, Natural Law and Thomist Philosophy of Law.
\end{abstract}

Key words: Philosophy of Law, Thomist Ethics, Natural Law.

\section{BREVE RESEÑA PERSONAL Y BIOGRÁFICAL ${ }^{1}$}

Rafael Fernández Concha nació en Santiago el 25 de octubre de 1833, el mismo año en que se puso en vigencia la Constitución que encarnaba el espíritu de Portales, y que traería enormes consecuencias para nuestra vida republicana, así como la obra del ilustre jurista estaría también destinada a señalar caminos en la historia intelectual de Chile. Su padre fue don Pedro Fernández Recio, abogado, profesor universitario y diputado. Participó en el tristemente célebre asunto conocido como la "cuestión del sacristán”, durante el gobierno de Montt, defendiendo a los canónigos Meneses y Solís de Ovando. En 1847 se le designó para suceder en la Facultad de Derecho de la Universidad de Chile a Mariano Egaña ${ }^{2}$. A la muerte de su esposa, en 1872, y siendo todos sus hijos mayores de edad, se ordenó sacerdote, recibiendo el presbiteriado en 1873, al cumplir 77 años. El ilustre hombre público fallecería diez años después de acceder a tal condición.

La madre del futuro filósofo del derecho y teólogo, doña Rosa de Santiago Concha y Jiménez Cerda, fue -según consta en los documentos de la época- mujer de gran

\footnotetext{
* Profesor de la Facultad de Derecho y de la Facultad de Filosofía, Pontificia Universidad Católica de Chile.

${ }^{1}$ Me baso, para esta información, en dos tesis de licenciatura de la Universidad de Chile, que abordan la biografía del ilustre jurista: Ayala (1947) y Quezada (1995).

${ }^{2}$ Universidad de Chile (1846) p. 45.
} 
santidad, de acuerdo a lo que atestiguan los cronistas que tuvieron contacto con ella ${ }^{3}$. Doña Rosa y don Pedro tuvieron trece hijos -circunstancia no poco usual en las familias santiaguinas de ese tiempo-, siendo los más conocidos Rafael y Domingo. Este último fue un personaje de la vida pública nacional: cuatro veces senador de la República, le cupo además directa participación económica en la fundación de la futura Pontificia Universidad Católica de Chile, en 1888. Fue, junto a lo mencionado, un pionero de la vitivinicultura en Chile, al hacerse asesorar por expertos franceses para traer a nuestro país las primeras cepas de ese origen en la Viña Santa Rita, que fundara en 1880 en la localidad de Alto Jahuel.

Don Rafael empezó en 1843, a los nueve años de edad, sus estudios en el Instituto Nacional, donde fue alumno de Bello y obtuvo las máximas calificaciones. Continuó posteriormente su educación en la Universidad de Chile, titulándose como Licenciado en Leyes en 1855, con una memoria sobre "La facultad de testar y el artículo 1354 del Proyecto del Código Civil”. El título de abogado le fue concedido en 1856.

Ayala atribuye a Carlos Casanueva la afirmación de que (Fernández Concha) "estaba dotado del más extraordinario talento especultativo que jamás haya habido en Chile" 4 . De acuerdo con la información que se extrae de cronistas como Vicente García Huidobro y Crescente Errázuriz, debe de haber sido un hombre de gran capacidad contemplativa, lo que le llevaba a abstraerse de improviso en medio de las reuniones, o manifestar desprecio por los avatares y circunstancias de la vida cotidiana, ya fuera pública o privada. Esto lleva a Errázuriz a calificarlo de un mal consejero (en asuntos ordinarios) y mal gobernante (es decir, jefe): "demasiado apegado a sus ideas, poco práctico, en exceso menospreciador de la opinión pública y nada conocedor de los hombres, porque casi no vivía en el mundo real, estaba en cada instante a punto de equivocarse y su error podía ser tanto o más funesto cuanto más notables eran sus cualidades de inteligencia y corazón y cuanto mayor tenacidad ponía en mantener sus opiniones" 5 . A la hora de apreciar esta semblanza, debe tenerse presente que Rafael Fernández Concha y el Arzobispo Errázuriz habían mantenido una ácida disputa cuando este último aceptó, en 1874, la dirección de "El estandarte Católico", pues en opinión del primero no se debía mezclar religión y política contingente.

Don Rafael era un hombre reservado y discreto, más bien serio y algo severo, muy alejado de cualquier forma de ostentación o preocupación por las cosas adventicias. Se trataba probablemente de un carácter retraído, orientado más a la reflexión y la oración que a la vida social, lo que lleva a Joaquín Yrarrázaval Larraín a decir que "vivía en la

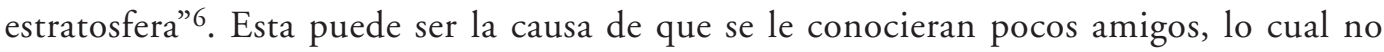
quiere decir que careciera de un corazón afectuoso o de sensibilidad, sino solo habla de

\footnotetext{
${ }^{3}$ Cruz Ugalde p. 6.

${ }^{4}$ Ayala (1947) p. 17. Encuesta hecha a Monseñor Casanueva, sin citar la fuente.

${ }^{5}$ Errázuriz (1934) p. 175.

${ }^{6}$ Yrarrázaval (1946) p. 175
} 
la fuerte vocación especulativa y de contemplación que se traduciría luego brillantemente en sus obras. Errázuriz lo tilda de "rígido". Es posible que su personalidad, por frecuentar los modelos éticos y jurídicos en su abstracción, pudiera haber resultado inflexible en un primer momento (aunque sus modales eran corteses hasta lo exagerado), pero nada lleva a pensar que un hombre cuya inteligencia era capaz de penetrar tan hondamente en la esencia del bonum faciendum et malum vitandum fuera al mismo tiempo incapaz de ajustar los principios universales a las circunstancias concretas. La imputación de rigidez pudiera aplicarse con mayor propiedad justamente a aquel modelo humano que Rafael Fernández Concha justamente no era: un formalista.

Desde el punto de vista de su aspecto, se ha dicho de él que "poseía una atrayente fealdad"7: era extremadamente alto y delgado, y su cabeza, irónicamente, resultaba muy pequeña en el contexto general de su cuerpo, como si la naturaleza hubiera resuelto manifestar hasta qué punto era grandiosa, poniendo tanto dentro de tan poco. Debe de haberse tratado de un tipo físico ascético y distante, no tanto por su desdén hacia las personas como debido a su inveterada abstracción, cuestión que probablemente se percibía en la mirada, tornándola fría más allá de lo que era en realidad.

En 1856 fue nombrado profesor suplente de Derecho Canónico en la Universidad de Chile, y en 1863 se le designa ya en propiedad, en virtud de sus indiscutibles méritos académicos. Aunque ejerció un tiempo la profesión, en 1859 ingresó al Seminario Conciliar. Al reemplazar a Antonio García Reyes en la Facultad de Leyes y Ciencias Políticas (1857), leyó su famoso discurso "Estudios jurídicos", al que nos referiremos más adelante. Hubo en aquella época una moción para suprimir la enseñanza del Derecho Romano y del español. Rafael Fernández Concha defiende ambos con gran vehemencia, y se transige en la pérdida solo del segundo.

En 1860 fue ordenado sacerdote, desempeñando innumerables cargos en su carrera eclesiástica y civil: fue nombrado diputado en 1870 y figuró en el Congreso Constituyente de ese mismo año. Además, el presidente Riesco lo nombró consejero de Estado, en 1901. No deja de llamar la atención cómo una persona supuestamente carente de las condiciones necesarias para el gobierno fue nombrado en cargos descollantes de prudencia durante toda su vida. Probablemente, esta fuera una más de las ambivalencias y contradicciones que encontramos en su vida y personalidad, la que, como se ha visto, no puede ser conocida sino por testimonios de terceros, pues no existen textos personales en los que hable de sí mismo. Aparentemente, tuvo una crisis espiritual de difícil datación, pero que puede situarse entre los años 1855 y 1857 fruto probablemente de su espíritu extraordinariamente crítico y de las influencias laicistas que quitarían al mundo católico personalidades como las de Barros Arana y Balmaceda. Parece haber sido, en su caso, un hecho puramente interior, que se conoció una vez superada. Puede ser que la causa de esta duda religiosa fue la lectura de las obras de Kant, pues cuando se refiere a las desviaciones del pensamiento, es su ejemplo más citado y más criticado. Esto, sin embargo, no pasa el plano de una mera hipótesis.

\footnotetext{
${ }^{7}$ Fernández de García Huidobro p. 24.
} 
Fruto de una rara enfermedad que solía aquejar a su familia, murió en Santiago, el 13 de octubre de 1912, a los 79 años de edad, sin tener ya conocimiento de lo que ocurría en el mundo exterior, al que tan poca importancia había concedido durante su vida dedicada a la reflexión.

\section{EL CONJUNTO DE SU OBRA INTELECTUAL}

Sin perjuicio de una apreciable variedad de artículos y obras menores (cartas, discursos, artículos periodísticos, memorias, documentos oficiales, etc.), la producción intelectual de Rafael Fernández Concha gira en torno a cuatro grandes obras, que marcan los pilares de su pensamiento: Derecho Público Eclesiástico, en dos volúmenes, publicado en 1872; Filosofía del Derecho o Derecho Natural, también en dos volúmenes, publicado en 1881; la Teología Mistica, que vio la luz en Barcelona durante el año 1889, y Del hombre en el orden psicológico, en el religioso y en el social, que apareciera en 1900.

Antes de comentar brevemente estos trabajos, conviene señalar que en su memoria de Licenciatura sobre la Facultad de Testar trata sobre la legítima ${ }^{8}$, y refuta a Vino estableciendo que la facultad de testar es creación de la ley civil, que exige que el ser humano deje reemplazada su persona jurídica. Refuta, además, la facultad omnímoda de testar, en contra de Bello, que dejaba a la voluntad del testador la distribución de la herencia. Fernández Concha indica que el Derecho tiene la obligación de limitar las libertades del amor de acuerdo con la razón. Defiende, además, contra Bello, el desheredar por causas justas, y refuta sus argumentos contra la facultad restringida de testar. Como dice Hanisch, "admira que un alumno de veintitrés años refute a Bello con tanta soltura, lo acusa de un error psicológico en sus apreciaciones y el comentario de Bello lo encuentra una pieza literaria, no jurídica" 9 .

El texto sobre Derecho Público Eclesiástico. Su origen fue un conjunto de artículos publicados en la Revista Católica durante el año 1872, escritos para influir en contra de las posiciones liberales, pero que son refundidos y mejorados de cara a la edición como libro. Este texto tuvo una segunda edición en 1894, donde el autor suprime sus comentarios sobre la libertad de enseñanza en Chile. Desde el punto de vista teórico, es interesante referirse al capítulo VIII de este trabajo, que versa sobre la moral, anunciando los temas filosóficos que desarrollará posteriormente en otras obras. Explica, con una apariencia voluntarista que la moral ha de consistir en el amor a Dios ${ }^{10}$, lo cual aclara casi de inmediato al afirmar que "si en cuanto seres racionales Dios es nuestro último fin, es claro que para obrar racionalmente, debemos dirigir hacia Él nuestras operaciones"11. Es decir, se ama a Dios en cuanto perfecto, y por ello es racional hacerlo el objeto de la voluntad. Y a continuación desarrolla los argumentos en contra de lo que llama la "moral independiente": aquella "opinión moderna" que separa la moral de la religión ${ }^{12}$, es decir, de lo que hoy

\footnotetext{
${ }^{8}$ Para lo que sigue, véase Hanisch (1970) p. 417

${ }^{9}$ Hanisch (1970) p. 417

${ }^{10}$ Fernández (1872) p. 124.

${ }^{11}$ Fernández (1872) p. 126.
} 
llamaríamos el fundamento trascendente del orden moral objetivo. Esta obra no tiene demasiada importancia para constatar su evolución del suarismo al tomismo, aunque ya indica aquí que la aprehensión de los singulares es indirecta, siguiendo al Aquinate, y separándose de Suárez sin mencionarlo explícitamente ${ }^{13}$.

Por su parte, la Teología Mistica es un tratado verdaderamente magistral que supone y demuestra un conocimiento exhaustivo de los autores más relevantes sobre la unión con Dios por la contemplación ${ }^{14}$; pero también de su experiencia personal. Fernández Concha solo trata de la cuestión más fundamental: la esencia y naturaleza de la contemplación infusa. Existía una segunda parte que nunca llegó a publicarse, denominada Teología mística práctica. Lo característico de su trabajo fue el enorme empeño desplegado para descifrar las cuestiones más difíciles con un lenguaje escolástico, lo que le llevaba a corregir las expresiones poéticas de algunos místicos españoles ${ }^{15}$. En este texto ya aparece casi completa su evolución hacia el tomismo, lo que no se muestra únicamente por la profusión de textos de Santo Tomás, sino especialmente, como dice Jiménez, en el hecho de que la verdadera inspiración fundamental y decisiva de toda su obra, tanto en las grandes líneas como en los pequeños problemas doctrinales, se encuentran orgánicamente subordinadas a un tomismo genuino y bien asimilado, que la proporciona las claves para enfrentar los problemas místicos ${ }^{16}$.

Su última obra con forma de libro, Del hombre en el orden psicológico, en el religioso y en el social, constituye un trabajo inmenso, de más de mil cuatrocientas páginas, cuyo objeto es, como lo sugiere el mismo censor eclesiástico don Rafael Vergara Antúnez, defender la fe y la moral objetiva en un mundo en el que la lucha contra el catolicismo se había situado en el terreno de la razón y de la ciencia. Se trataba, pues, de entregar al lector culto un conjunto de conocimientos antropológicos y filosóficos destinados a efectuar una apologética capaz de detener los impulsos del liberalismo y del laicismo. El mismo Fernández Concha lo declara de este modo, al afirmar que "hice este libro con el fin de atraer a la religión a caballeros de cierta edad, a hombres ya formados, de estudios, a intelectuales" ${ }^{17}$. El censor alaba "el servicio que ha de prestar a los hombres de fe vacilante”. Como su nombre lo indica, está dividido entres partes: un tratado del alma en general y su unión con el cuerpo; un tratado sobre Dios, la Revelación y la Iglesia, y un tratado final sobre las sociedades doméstica, civil y religiosa. Se trata de un texto que se inscribe en la más ortodoxa tradición tomista.

\section{EL DERECHO, LA FILOSOFÍA DEL DERECHO Y EL DERECHO NATURAL}

En su texto de 1857 sobre la enseñanza del Derecho, Fernández Concha viene a proponer lo que él consideraba una mejor organización de los estudios jurídicos, separando su discurso en tres partes:

\footnotetext{
12 Fernández (1872) pp. 130 ss.

13 Fernández (1872) p. 82.

${ }^{14}$ Los más citados son Santo Tomás y San Juan de la Cruz.

15 Hanisch (1970) p. 452.

16 Jiménez (1962) pp. 524-5.

17 Ayala (1947) p. 53, tomado de la encuesta a don Vicente García Huidobro.
} 
a) La determinación de las especialidades en que debe dividirse una ciencia.

b) El orden metódico en que ha de disponerse su enseñanza, y

c) La manera de tratar esta enseñanza.

El núcleo del discurso es el siguiente: afirma que lo primero en el conocimiento de un saber es la comprensión de todas las nociones que dicen con el orden de ideas que la constituye. Y se queja de que, en los seis años de carrera, no se proporcionan a los alumnos ideas sobre los principios del Derecho: su lugar entre las ciencias, su organización y geografía, los criterios sobre lo justo o injusto. Es decir, está solicitando un curso de Introducción al Derecho. Y propone reemplazar la asignatura de Derecho Natural por este $^{18}$, porque se había convertido en una mera crítica de las instituciones sociales, abandonando su objeto propio. Sostiene que el jurista debe cultivar su saber en las fuentes de la filosofía, partiendo desde la idea misma de Derecho (que en otro texto posterior definirá como aquella parte de la moral cuyo cumplimiento no es posible dejar a la mera conciencia de los obligados por el deber, cuyo cumplimiento demanda el bien social que se haga efectivo por la fuerza pública ${ }^{19}$ ). Después, es necesario ordenar científicamente cada especialidad, para que brille la unidad en torno a un solo centro de gravitación. La preeminencia de este centro, la centralidad de la esencia de las cosas (es decir, la contemplación de los principios y la buena organización de las áreas regionales), conseguirá, piensa Fernández Concha, que el jurista sea capaz de unir la historicidad del Derecho con la justicia inmanente en la naturaleza. Esta es también la causa por la que insiste en la necesidad de los estudios histórico-jurídicos como parte del programa de la carrera.

El texto es una crítica al currículum de 1853, que solo buscaba entregar conocimientos prácticos o profesionales a los abogados. Esta preocupación filosófica, como dice Baeza, lo acerca a Lastarria ${ }^{20}$. Es posible que la incorporación del curso de Introducción al Derecho se deba a este texto de RFC. Lo que resulta indudable es su posterior influencia, pues es reeditado y citado en muchas ocasiones en los manuales introductorios.

Hemos dejado para el final la Filosofía del Derecho, su gran obra, precisamente porque esta fue escrita con ese ánimo introductorio que se declara en el discurso que acabamos de revisar, y tal como lo declara su mismo subtítulo: "dispuesto para servir de introducción a las ciencias legales". El origen del libro se encuentra en el discurso de ingreso a la Facultad de Leyes, donde sienta los principios de su tesis sobre la introducción al Derecho. Posteriormente, publica en la Revista Católica (1874) un conjunto de quince artículos anónimos, con los temas que contendría la obra, cuya publicación aconteció en 1881. La segunda edición tuvo lugar en Barcelona en 1887 y 1888, también en dos tomos. Fue reeditado por tercera vez al cumplirse el cincuentenario de la muerte del autor, y, como lo expresa Hübner Gallo en el prólogo de la edición de 1965, se trata "de un magistral tratado...una obra clásica en la bibliografía jurídica hispano-

\footnotetext{
18 Fernández (1857) p. 133.

19 Fernández (1872) p. 142.

20 Baeza (1944) p. 141.
} 
americana... el más valioso tratado que se haya publicado sobre la materia en el país, y uno de los más importantes que se han escrito en nuestro idioma" ${ }^{21}$. El primer texto sobre materias teórico-jurídicas que se publicó en Chile fue el de José Joaquín de Mora, que vio la luz en 1830, y su objeto, más que realizar grandes especulaciones, fue el de satisfacer necesidades docentes impostergables. Andrés Bello también enseñó cuestiones relativas al Derecho Natural, pero no dejó casi nada escrito, y desde luego su orientación doctrinal contradecía el punto de partida fundamental que suscribía Rafael Fernández Concha $^{22}$.

Para comprender bien el sentido de esta obra en el contexto intelectual del eminente jurista, es preciso tener en cuenta el proceso de su evolución intelectual. Fernández Concha, quien sostuvo en un principio una orientación filosófica de corte suarista, fue variando su posición hasta hacerse completamente tomista, cuestión que se refleja exhaustivamente en la obra que comentamos. Crescente Errázuriz dice de él que después de haber sido por mucho tiempo "partidario de Suárez, con cuyas obras se le veía de continuo en las manos, y en donde buscaba solución a cualquier dificultad... a medida que se vio obligado a estudiar en la fuente, en Santo Tomás, algunas cuestiones; y al cabo su conversión llegó a ser total, absoluta, y su tomismo más entusiasta aún de lo que había sido su molinismo"23. Este proceso no se limitó a las obras del Aquinate, sino también abarcó a sus comentadores, como lo demuestra la erudición y amplitud que el autor exhibe en sus debates filosóficos.

¿Cómo se produjo este cambio de orientación? Debe tenerse en cuenta que, hasta el momento en que ingresa al Seminario, sus conocimientos de la filosofía se reducen a materias relativas a la filosofía que hoy llamaríamos "moderna", es decir, aquella que le era contemporánea. Sin perjuicio de que existen referencias a Santo Tomás de Aquino en sus primeros trabajos, ello no demuestra un conocimiento en profundidad, sino solo un cierto barniz, producto de una tradición que en esa época ya empezaba a hacer crisis. Sin embargo, su ingreso al Seminario le lleva a encontrarse directamente con los textos escolásticos, sistema de pensamiento que le produjo una fuerte conmoción, probablemente por la concepción unitaria y ordenada del mundo, que ofrece, y que Fernández Concha buscaba más allá de las modas intelectuales. Dentro de este contexto, su inclinación inicial a Suárez parece haber sido exclusivamente fortuita, y como tal desapareció en cuanto descubrió en otros textos los sólidos fundamentos que buscaba. Crescente Errázuriz transcribe la siguiente anécdota: el Arzobispo Casanova había, por aquel entonces, encargado estudiar un catecismo a Jorge Montes. En medio de la discusión, Montes se apoyó en Suárez para defender una opinión, a lo que nuestro autor respondió con un gesto que indicaba que le daba poco valor. Entonces, Montes acoto: "pero hombre, ¿no fuiste tú su admirador entusiasta durante muchos años? Sí -respondió Fernández Concha-; bastante tiempo perdî" 24 . Hanisch reafirma lo que apuntábamos antes, en el sentido de que ya en la Teología

\footnotetext{
${ }^{21}$ Hübner (1965) pp. IX-X.

22 Hübner (1965) p. XI.

${ }^{23}$ Errázuriz (1934) p. 172.

${ }^{24}$ Errázuriz (1934) p. 173.
} 
Mistica el cambio de orientación intelectual se había producido, pues "ensaya nuevas formas literarias no usadas antes por él”, y cita numerosos pasajes tributarios de la obra aquinatense ${ }^{25}$.

La obra de Rafael Fernández Concha no es exactamente lo que hoy se denominaría una "Filosofía del Derecho", sino más bien un tratado de Derecho Natural, con elementos de psicología filosófica y ética tomista. El texto se abre con una introducción que, como indicaba en su discurso de incorporación a la Facultad de Derecho, sitúa la ciencia en su contexto epistemológico, exhibe su importancia y justifica el método. Su primer tema sistemático es el acto humano, comenzando por una brillante explicación sobre el bien y la libertad. Este texto posee una claridad expositiva y argumental tan descollante, que con dificultad puede encontrarse nada parecido entre los múltiples seguidores del Santo de Aquino. Después de referirse a la imputabilidad, la moral y la ley natural, Fernández Concha entra de lleno en el Derecho, explicando su esencia y propiedades. Opta claramente por la primacía del derecho objetivo, al afirmar que "para formarse cabal idea de la esencia del Derecho no basta saber que este envuelve una relación entre dos o más personas, resultante del poder de obrar, lícito e inviolable, que compete a una, y de la necesidad moral que pesa sobre otras, de no atacar el ejercicio de dicha facultad. Es necesario saber además cuál es la materia u objeto sobre que recae la referida relación, pues solo ella puede darnos a conocer la existencia de un derecho" 26 . Esta primacía del derecho objetivo es, a fin de cuentas, la primacía de la ley que se funda en la naturaleza y que es descubierta por la razón, por cuanto es en virtud de la obligación que esta nos impone de obrar conforme a los dictados de la razón que nos encontramos sujetos a obedecer ${ }^{27}$.

Especial interés reviste, en nuestra opinión, su explicación de la epiqueia, por su claridad y rigor. Dice Fernández Concha que "se entiende por epiqueia una especial interpretación de la ley, por la cual se declara que un caso particular, en razón a las peculiares y extraordinarias circunstancias del mismo, no se halla comprendido en la disposición del Derecho, no obstante ser generales y conspicuos los términos de ella"28. Esto supone el reconocimiento de que la ley humana es naturalmente imperfecta, por su brevedad y generalidad, e incapaz en consecuencia de hacerse cargo de todos los casos posibles. He aquí el corazón del iusnaturalismo, en contra del racionalismo y el formalismo jurídico.

Hay una extensa crítica a los sistemas relativos a la esencia de la moralidad, contrastándolos con el pensamiento tomista; así como una reflexión sobre la distinción entre la moral y el Derecho, en el mismo sentido. Esta parte del trabajo demuestra el profundo conocimiento que tenía de las doctrinas modernas de su época. El que el curso de Derecho Natural que se impartía contuviera solo estas críticas y diferencias con las tesis contemporáneas era, como se explicaba antes, lo que llevaba a Fernández Concha a

\footnotetext{
${ }^{25}$ Hanisch (1970) p. 425, nota 4.

26 Fernández (1872) p. 91.

${ }^{27}$ Fernández (1872) p. 116.

${ }^{28}$ Fernández (1872) p. 149.
} 
buscar suprimirlo. Desde el punto de vista de la esencia de la moral, la conclusión de nuestro autor es que el objeto general de la ética es la recta ordenación de los actos libres, de la cual proviene en ellos la bondad moral. Al dividirse dicho objeto general en varios particulares, se deducen las distintas especies de bondad moral que se encuentran en las acciones libres. Entre esas especies distintas de bondad moral se halla la justicia, y la ciencia que trata de la justicia es el Derecho. "Según resulta de lo dicho, la ciencia jurídica no es más que una rama de la ciencia ética" 29.

Ya en el volumen segundo de su obra, Fernández Concha se dedica en primer lugar al derecho individual, sea innato o adquirido. Dentro de los primeros, describe el derecho de personalidad, de libertad o independencia, de asociación, de vida y de defensa. Particularmente sobresaliente nos parece la explicación que efectúa de estos dos últimos. Define el derecho de vida como "el que tenemos a que nadie atente contra la nuestra" ${ }^{30}$, describiéndolo como un "derecho indirecto", no consiste en el dominio sobre ella, sino solo en la facultad de exigir de los otros su inviolabilidad. Esta ausencia de dominio pleno sobre la propia vida desautoriza también el suicidio, cuestión en la que sigue, nuevamente, la posición tomista, que resuelve un problema muy debatido desde el punto de vista histórico. Por ejemplo: San Atanasio lo condena como una cuestión de principios. San Gregorio Nacianceno alaba algunos casos del Antiguo Testamento, pero lo condena en general, como San Ambrosio. San Jerónimo es incoherente, pues lo rechaza en los cristianos pero alaba a las viudas paganas que lo prefieren a volver a casarse. Orígenes y Dionisio, por su parte, afirmaban que Jesús se suicidó, pero consideran que no cabe exponerse a él innecesariamente. El único que es tajante en la condena es San Clemente de Alejandría, sosteniendo que los cristianos que se suicidan tienen una concepción errónea del martirio. Los teólogos moralistas del s. IV suelen seguir las tesis neoplatónicas: lo condenan en principio pero admiten excepciones, como cumplir una orden de la autoridad (Sócrates), evitar la vergüenza o un destino cruel. Como se ve, la doctrina estaba muy poco clara. En el año 348 el Concilio de Cartago lo condenó, contra los donatistas, que lo defendían, y en 381, el Obispo de Alejandría decretó que no se podían decir oraciones por los suicidas, excepto en los casos de locura debidamente certificada. Quien declara lo que sería la posición oficial de la Iglesia es San Agustín. El que se suicida es un cobarde incapaz de afrontar la adversidad. Es la vanidad la que lo induce, al dar importancia a lo que los otros piensan de uno. Ninguna circunstancia lo excusa, ni siquiera la violación. La posición adversa al suicidio se endurece desde el s. V, en contra de la posición romana, más indulgente, hasta derivar en la posición de Santo Tomás, que Fernández Concha reproduce al afirmar que "tal atentado nunca es lícito: no hay ni puede haber circunstancia, aun entre las más extraordinarias, capaz de justificarlo ni excusarlo"31.

En cuanto al derecho de defensa, nuestro autor parte del principio de que nunca se puede dar muerte al inocente, situación que no se presenta frente a la agresión ilegítima, frente al que surge la posibilidad jurídica de esta defensa, que consiste solo en

\footnotetext{
${ }^{29}$ Fernández (1872) pp. 335-6.

30 Fernández (1872) vol. II, p. 28.

31 Fernández (1872) p. 29.
} 
"dejar incólume el derecho atacado, impidiendo y destruyendo la agresión" (de donde se deduce que no es un acto de venganza ${ }^{32}$. La conclusión de nuestro autor es que a veces es lícito hacer daño al prójimo -incluso hasta tomar su vida-, cuando en ello consiste la única defensa contra el ataque a nuestra persona o a nuestros bienes, y siempre que esa defensa sea proporcionada a la agresión y cese en cuanto termine la posibilidad cierta del daño actual o inminente.

Dentro de este mismo volumen, trata Fernández Concha de la propiedad, en cuanto derecho adquirido, y de la sociedad conyugal, política y religiosa -temas que volverá revisar después en su texto sobre el hombre-, con una perspectiva más filosófica y menos jurídica que la presente. El resto del trabajo, fiel a su intención declarada mucho antes, de proporcionar al alumno una visión ordenada, jerárquica y unitaria de las áreas regionales del Derecho, está dedicado a las partes especiales de la ciencia: Derecho Administrativo, Penal, Procesal e Internacional o de Gentes.

De todo lo dicho, puede deducirse que, en el contexto del reducido espacio de tiempo que los países iberoamericanos pueden exhibir como naciones soberanas e independientes, la personalidad intelectual de Fernández Concha es una de las más notables que Chile ha visto nacer; país tan fértil en historiadores, cronistas, poetas, e incluso, mutatis mutandis, juristas, pero desde luego no en grandes mentes especulativas. Este es, justamente, el gran mérito del pensador cuya vida y obra comentamos. La notable penetración intelectual, unida a una sorprendentemente clara e hilada argumentación, hacen de sus textos piezas casi únicas en la historiografía de la inteligencia nacional. El impulso que su trabajo da a la filosofía escolástica, y específicamente en los estudios ético-jurídicos, ha desempeñado además un factor relevante no solo en la composición de los programas de las Facultades de Derecho en las dos grandes universidades del país en los siguientes cincuenta años, sino en la impronta intelectual de claridad y lucidez que sus escritos desbordan hasta el día de hoy, cuyo lenguaje -podría pensarse- se encontraría pasado de moda. Pensamos que, desde el punto de vista de la escolástica tomista en Chile, la figura de Rafael Fernández Concha solo es comparable a la de un contemporáneo, el padre Osvaldo Lira, SSCC, cuyos estudios filosóficos excedían también el campo estrictamente jurídico, con idéntica lucidez y capacidad comprensiva.

La rigurosidad, la gran erudición y la exactitud argumental que se observa en las obras de Rafael Fernández Concha basta por sí sola para convertirlo en figura señera dentro del mundo de habla hispana, al menos en el campo de la llamada philosophia perennis. En este sentido, es de lamentar que sus obras no hayan vuelto a ser editadas; esto es una tarea pendiente en la revalorización de nuestra tradición intelectual.

Fecha de recepción: 30 de marzo de 2006

Fecha de aceptación: 28 de abril de 2006

32 Fernández (1872) p. 32-3. 


\section{BIBLIOGRAFÍA CITADA}

Ayala Godoy, Hugo (1947): Rafael Fernández Concha (Talleres Gráficos "El Chileno", Santiago).

Baeza Marambio, Mario (1944): Historia de la Facultad de Ciencias Jurídicas y Sociales de la Universidad de Chile (Imprenta Universitaria, Santiago).

CRuz Ugalde: Flor de Chile. Pro Manuscripto.

ERrázuriz, Crescente (1934): Algo de lo que he visto (Santiago, Nascimento) pp. 476.

Fernández de García Huidobro, María Luisa: "El Tío Juan", en Revista Aliados, año 1 n. 10.

Fernández Concha, Rafael (1872): Derecho Público Eclesiástico (Santiago, Imprenta del Correo).

FERnÁNDEZ CONCHA, Rafael (1857): "Estudios Jurídicos”, en: “Anales de la Universidad de Chile, vol. XIV.

FERNÁNDEZ CONCHA, Rafael (1966): Filosofía del Derecho, vol. I (Santiago, Ed. Jurídica de Chile).

JiméneZ, Julio, S.J. (1962): Rafael Fernández Concha y su Teología Mistica (Roma).

HanisCH, Walter, S.J. (1970): "Rafael Fernández Concha y su Teología Mística”, en: Estudios en Honor de Pedro Lira Urquieta (Santiago).

HÜBner Gallo, Jorge Iván (1965): "Prólogo", en: Fernández Concha, Filosofía del Derecho"(Editorial Jurídica de Chile, Santiago).

QueZada NúNEEZ, Jorge (1995): Juristas chilenos del s. XIX: Rafael Fernández Concha, pro manuscripto.

Universidad de Chile (1846): Anales de la Universidad de Chile, sec. 3.

YrarráZaVAl Larraín, Joaquín (1946): Para mis hijos (Santiago). 
